\title{
Contributions of sleep physiology to empathic capability in helping people
}

\author{
Makoto Uchiyama ${ }^{1,2}$
}

Published online: 2 January 2021

(c) Japanese Society of Sleep Research 2021

Empathy is defined as an ability to place oneself into another person's situation. A current advance in psychology has proposed that human empathy is composed of cognitive empathy and affective empathy, both of which are different in formal aspects but equally important for helping one form and maintain connections with others [1]. Since sincere and properly expressed empathy toward others in trouble develops and nurtures interpersonal connections, leading to optimization of psychological defense and enhancement of positive energy [2]. Therefore, functions of empathy have been regarded as among crucial factors for optimal interpersonal relationships in various medical services [3].

Empathy in a patient-physician relationship is considered important and beneficial in both directions. Patients who perceive their physicians as empathetic experience greater satisfaction and are more compliant with physician recommendations, while physicians experience greater job satisfaction, which may be protective against burnout [3]. Works of empathy in the service providers have at least two domains, including both affective and cognitive ones. Cognitive empathy is the ability to accurately identify the patients' emotional states, while affective empathy is the ability to experience the patients' emotional conditions [3]. A recent preliminary report has pointed out that sleep deprivation can disturb affective empathy among paramedics, which might provide serious negative influences on professional medical activities and stressed extensive research on such effects of empathy [4]. However, there are scanty findings or reports in terms of empathy in practical medical services.

Makoto Uchiyama

uchiyama.makoto@nihon-u.ac.jp

1 Tokyo Adachi Hospital, Hokima, Adachi, Tokyo 121-0064, Japan

2 Department of Psychiatry, Nihon University School of Medicine, Oyaguchi-Kamicho, Itabashi, Tokyo 173-8610, Japan
In the present issue, Amalean et al. [5] aimed to see whether sleepiness might have a detrimental effect on cognitive empathy in emergency medical technicians as had been shown in affective empathy and examined their empathy differential from cognitive and affective aspects prior to and following working a 12-h day or night EMT shift. They used the Reading the Mind in the Eyes (RMET) task and the Toronto Empathy Questionnaire (TEQ) for cognitive empathy and affective empathy, respectively. The comparison between pre-shift and post-shift empathy scores demonstrated that affective empathy did not differ before and after a 12-h shift, while cognitive empathy did decrease after a 12-h shift and was thus affected by shift work.

Though there were several methodological limitations in the present study, including a relatively small sample size and somewhat unsophisticated points in the study design, it is worthy of being published in Sleep and Biological Rhythms as it is the first study to suggest that different forms of empathy have been differentially influenced by circadian timing and homeostatic build-up of sleepiness, both of which have fundamental biological significance in the control of mammalian sleep-wake cycle. These findings also seem to indicate that such different forms of empathy may have different neurophysiological bases.

\section{Compliance with ethical standards}

Conflict of interest The author has received research support from Astellas Pharma, Eisai, Meiji Seika Pharma, MSD, Taisho Pharmaceutical, Kao Corporation, and Takeda Pharmaceutical, and has consulted for Idorsia Pharmaceuticals Japan, Kao Corporation, Taisho Pharmaceutical and Takeda Pharmaceutical. He has also received honoraria for giving lectures and/or contributing text from Eisai, Meiji Seika Pharma, MSD, and Takeda Pharmaceutical. 


\section{References}

1. Davis MH. Measuring individual differences in empathy; evidence for a multidimensional approach. J Pers Soc Psychol. 1983;44:113-26.

2. Decety J, Lamm C. Human empathy through the lens of social neuroscience. Sci World J. 2006;6:1146-63.

3. Lamothe M, Boujut E, Zenasni F, Sultan S. To be or not to be empathic: the combined role of empathic concern and perspective taking in understanding burnout in general practice. BMC Fam Pract. 2014;15:15.

4. Guadagni V, Cook E, Hart C, Burles F, Iaria G. Poor sleep quality affects empathic responses in experienced paramedics. Sleep Biol Rhythm. 2018;16:365-8.
5. Amalean A, Pavuluri H, Schmidt WM, Fowler LA. The effects of sleepiness, time of day, and 12-hour shifts on affective and cognitive empathy in emergency medical technicians. Sleep Biol Rhythms. 2020; p. 18.

Publisher's Note Springer Nature remains neutral with regard to jurisdictional claims in published maps and institutional affiliations. 